\title{
Characterized Microstructure and Electrical Properties of Hydrogenated Nanocrystalline Silicon Films by Raman and Electrical Conductivity Spectra
}

\author{
Gao XiaO-Yong $^{a, b, *}$, Zhao Jian-TaO ${ }^{a}$, Liu Yu-Fen ${ }^{a}$, Lin Qing-Geng ${ }^{a}$, \\ Chen Yong-Sheng ${ }^{a}$, Gu Jin-HuA ${ }^{a}$, Yang Shi-E ${ }^{a}$ And Lu Jing-XiaO ${ }^{a}$ \\ ${ }^{a}$ The Key Lab of Materials Physics of Ministry of Education, Zhengzhou University \\ 450052 Zhengzhou, China \\ ${ }^{b}$ School of Physics and Engineering, Zhengzhou University, 450052 Zhengzhou, China
}

(Received October 1, 2008; in final form October 21, 2008)

\begin{abstract}
Microstructure and electrical properties of hydrogenated nanocrystalline silicon (nc-Si:H) film deposited on glass substrate at low temperature were characterized by average grain size, crystallinity, and dark electrical conductivity data obtained from the Raman and electrical conductivity spectroscopy, respectively. The average grain size, crystallinity and electrical conductivity have a similar change with substrate temperature. A threshold substrate temperature determined by silane concentration appears in their corresponding spectroscopy vs. substrate temperature. The dependence of crystallinity, average grain size and electrical conductivity on substrate temperature were accounted for by surface diffusion model and heterojunction quantum dot model, respectively.
\end{abstract}

PACS numbers: 71.23.Cq, 73.61.-r

\section{Introduction}

More and more attention has been focused on hydrogenated nanocrystalline silicon (nc-Si:H) film because of its promising application to stable high efficiency solar cells [1-3], thin film transistors and panel display [4-6]. As a kind of candidate material, nc-Si:H has many special properties different from amorphous silicon such as higher electrical conductivity, smaller light-induced degradation and easy large-scale production. Its many applications for panel display, thin film resonance transistors and new optoelectrical sensor and detectors are based on its quantum size effects such as visible light photoluminescence [7] and tunneling effects [8]. On one hand, its microstructure determines its optoelectronic and electrical properties. On the other hand, silane concentration $\left(\left(\mathrm{SC}=\left[\mathrm{SiH}_{4}\right] /\left(\left[\mathrm{SiH}_{4}\right]+\left[\mathrm{H}_{2}\right]\right)\right)\right.$, substrate temperature $\left(T_{\mathrm{s}}\right)$ and other deposition parameters determine its microstructure. Generally, nc-Si:H is difficult to obtain due to the complex relationship between microstructure and deposition parameters. Based on it, its microstructure and electrical properties were characterized by average grain size, crystallinity and electrical conductivity data obtained from the Raman and electrical conductivity spectroscopy, respectively. The dependence of crystallinity, average grain size, and electrical conductivity on $T_{\mathrm{s}}$ were accounted for by surface diffusion model and heterojunction quantum dot (HQD) model, respectively.

* corresponding author; e-mail: xygao@zzu.edu.cn

\section{Experimental details}

A series of nc-Si:H films were deposited on glass substrate at different $\mathrm{SC}$ and $T_{\mathrm{S}}$ by using traditional radio frequency plasma-enhanced chemical vapor deposition (RF-PECVD) technique with an excitation frequency of 13.56 MHz. To avoid oxygen contamination, the base pressure in chamber is below $2 \times 10^{-4} \mathrm{~Pa}$ and working pressure keeps a high value of $133 \mathrm{~Pa}$ which can effectively lower the damage to sample surface by the ion bombardment. The film microstructure was characterized by average grain size and crystallinity obtained from the Raman spectroscopy and electrical properties characterized by dark electrical conductivity. The film thickness was determined from reflectivity spectra in terms of interference effects. All the measurements above were done at room temperature.

\section{Results and discussion}

\subsection{Mechanism of the film deposition}

The deposition rate of as-deposited nc-Si:H films is shown in Fig. 1 as a function of SC. The result indicates that the deposition rate is nearly linear with SC, which can be accounted for by the following reversible reaction:

$$
\mathrm{SiH}_{4}+\mathrm{e} \rightarrow \mathrm{SiH}_{4-m}+m \mathrm{H} \leftrightarrow \mathrm{Si}+4 \mathrm{H},
$$

where $m$ can be $1,2,3$, which is determined by electron energy [9]. For the threshold energy of producing $\mathrm{SiH}_{3}$, $\mathrm{SiH}_{2}$, and $\mathrm{SiH}$ is $8.75,9.47$, and $10.33 \mathrm{eV}$ [10], respectively, $\mathrm{SiH}_{3}$ is the main precursor radical beside $\mathrm{SiH}_{2}$ and $\mathrm{SiH}$. $\mathrm{SiH}_{4}$ reacts with plasma to produce active $\mathrm{SiH}_{4-m}$ and $\mathrm{SiH}_{4-m}$ decomposes into $\mathrm{Si}$. This reaction is positive reaction. At the same time, $\mathrm{H}$ can destroy the weak $\mathrm{Si}-\mathrm{Si}$ bonding and form active $\mathrm{SiH}_{4-m}$, which is reverse 


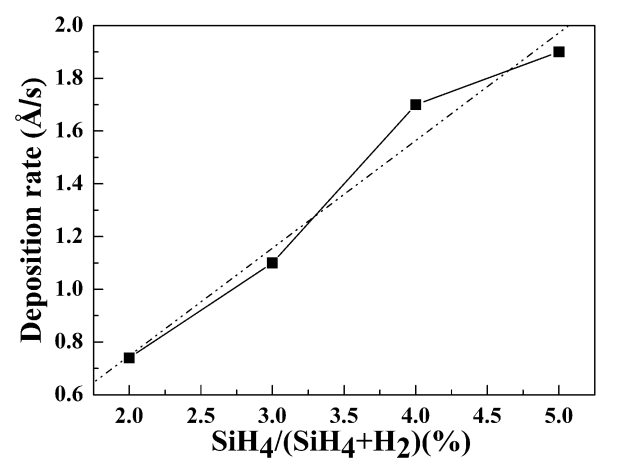

Fig. 1. Deposition rate of as-deposited nc-Si:H films as a function of silane concentration. The black squared and dash-dotted lines denote the measured and linear fitting line, respectively.

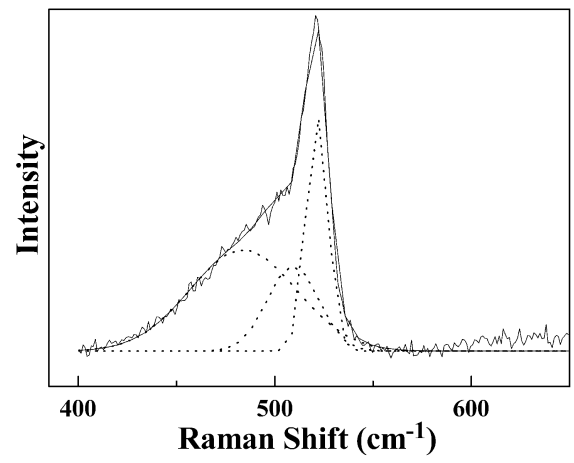

Fig. 2. Raman pattern for as-deposited nc-Si:H films.

reaction or called $\mathrm{H}$-situ etch reaction. In terms of the reversible reaction (1), the larger SC helps to enhance the positive reaction and restrain the reverse reaction. Hence, the deposition rate is nearly in proportion to SC.

\subsection{Characterization of the film microstructure}

The crystallinity of nc-Si:H film is investigated by the Raman spectroscopy. By using the fitting method, the transverse optical phonon mode spectroscopy is decom-

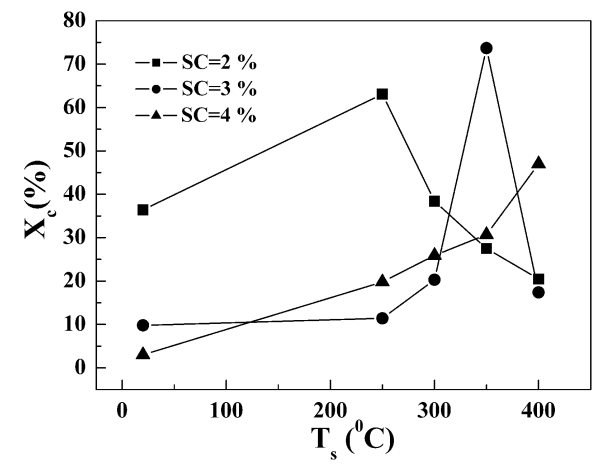

Fig. 3. Dependence of the crystallinity of as-deposited nc-Si:H films on substrate temperature. posed into three peaks, a crystalline peak $\left(520 \mathrm{~cm}^{-1}\right)$, an amorphous peak $\left(480 \mathrm{~cm}^{-1}\right)$ and an intermediate peak $\left(510 \mathrm{~cm}^{-1}\right)$. The Raman pattern of as-deposited nc-Si:H film is shown in Fig. 2. The dotted curves are the three-peak Gaussian fitting curves. The intermediate peak is considered to be due to grain boundaries, where increased bond lengths of the crystalline regions shift the Raman peaks to lower energy and to a grain size effect when the grain size is smaller than $15 \mathrm{~nm}$ [11]. The crystallinity for nc-Si:H can be described by formula $2[12,13]$ :

$$
X_{\mathrm{c}}=\left(I_{510}+I_{520}\right) /\left(I_{480}+I_{510}+I_{520}\right),
$$

where $I_{520}, I_{480}$, and $I_{510}$ are integrated intensities of the crystalline, amorphous and intermediate peak, respectively. The dependence of the crystallinity on $T_{\mathrm{s}}$ for nc-Si:H films is shown in Fig. 3. As can be seen from Fig. 3, the threshold $T_{\mathrm{s}}$ increases with $\mathrm{SC}$ increasing for $\mathrm{SC}$ smaller than $4 \%$. Conversely, no threshold $T_{\mathrm{s}}$ is observed in Fig. 3 for the as-deposited film at $4 \%$ SC. It implies that the determined threshold $T_{\mathrm{s}}$ is higher by $400^{\circ} \mathrm{C}$ for $4 \%$ SC. The results can be explained by surface diffusion model [14]. From Fig. 1, the deposition rate is nearly linear with SC. Therefore, the larger SC leads to a larger deposition rate, which makes the main reaction precursor $\mathrm{SiH}_{3}$ radical more difficult to migrate to lattice site by lattice relaxation method. On the other hand, the ratio of hydrogen coverage on film surface decreases with $\mathrm{SC}$ increasing. In terms of surface diffusion model, the main reaction precursor $\mathrm{SiH}_{3}$ radical firstly migrates to a crystalline Si site on the hydrogen-terminated Si surface and then is stabilized by bonding two surface Si atoms after releasing one $\mathrm{H}$ atom in about $200 \mathrm{ps}$ [15]. The smaller ratio of hydrogen coverage on film surface is, the more difficult $\mathrm{SiH}_{3}$ radical is to diffuse to a crystalline site by migrating toward the smaller hydrogen coverage ratio surface [14]. To realize crystallization, $\mathrm{SiH}_{3}$ radical has to migrate to crystalline $\mathrm{Si}$ site by the increase in kinetic energy resulting from the energy absorption from substrate surface. Hence, the larger SC is, the higher the threshold $T_{\mathrm{s}}$ of crystallization is. The crystallinity increases caused by the increasing kinetic energy and migration rate of $\mathrm{SiH}_{3}$ radical with $T_{\mathrm{s}}$ increasing until the

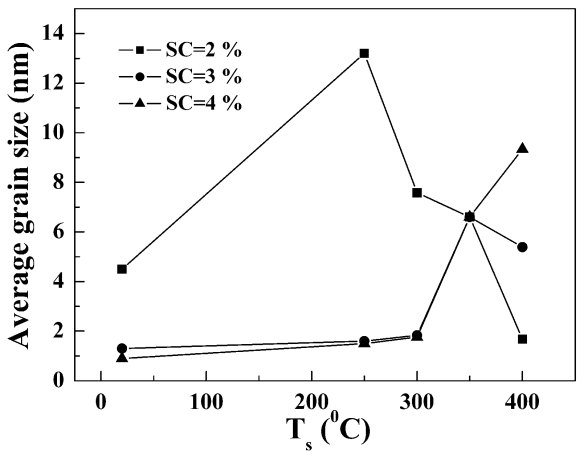

Fig. 4. Average grain size for as-deposited nc-Si:H films as a function of substrate temperature. 


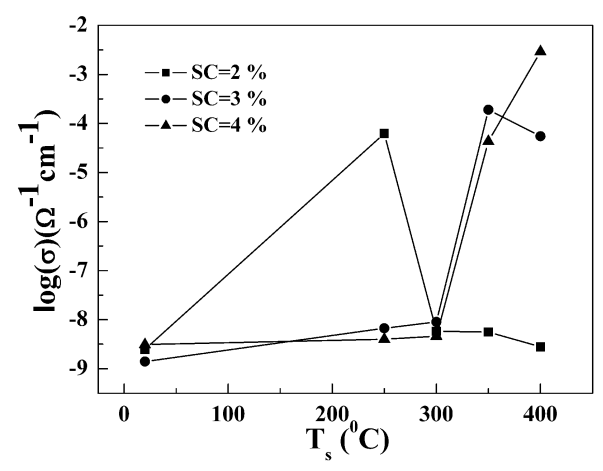

Fig. 5. Dark electrical conductivity of as-deposited nc-Si:H films as a function of substrate temperature.

threshold $T_{\mathrm{s}}$. However, the crystallinity decreases due to the hydrogen releasing from surface and $\mathrm{SiH}_{3}$ migration rate slowing down with $T_{\mathrm{s}}$ above the threshold $T_{\mathrm{s}}$. The hydrogen releasing can distort the ordered Si net and lowers the crystallinity. The average grain size $d$ for nc-Si:H film is shown in Fig. 4 as a function of $T_{\mathrm{s}}$. It is calculated in terms of the following formula:

$$
d=2 \pi(B / \omega)^{1 / 2},
$$

where the constant $B$ is $2.21 \mathrm{~nm}^{2} / \mathrm{cm}$ and $\omega$ is the deviation from the wave number of the standard crystalline silicon Raman peak. Figure 4 indicates a similar change between average grain size and crystallinity. It can also be explained by surface diffusion model. With $T_{\mathrm{s}}$ increasing, the main reaction precursor $\mathrm{SiH}_{3}$ radical easily migrates to a crystalline Si site on the hydrogen-terminated Si surface and bonds two surface Si atoms by releasing one $\mathrm{H}$ atom. The stabilized $\mathrm{Si}$ grain size can become large due to the stack of $\mathrm{Si}$ atoms. However, when $T_{\mathrm{s}}$ is higher than the threshold $T_{\mathrm{s}}$ of crystallization, surface $\mathrm{H}$ atom can release from surface and distort the stabilized Si net, so the grain size can become smaller.

\subsection{Characterization of the film conductivity}

The dark electrical conductivity of as-deposited nc-Si:H samples is shown in Fig. 5 as a function of $T_{\mathrm{S}}$. Figure 5 indicates that the electrical conductivity obviously changes in the range of $10^{-2}$ to $10^{-5} \mathrm{~S} / \mathrm{cm}$ with $T_{\mathrm{S}}$. The threshold $T_{\mathrm{s}}$ for electrical conductivity also appears in Fig. 5 implying a close relationship with crystallinity and average grain size. In terms of classical electrical theory, electrical conductivity is determined by free carrier concentration and mobility. However, as a kind of composite material made up of amorphous phase, grain boundary and nanocrystalline phase, the transport mechanism of nc-Si:H film is complex. In terms of heterojunction quantum dot model [16], nc-Si:H film is composed of alternating amorphous and microcrystalline regions, where the crystallinity keeps about 50\%. Between amorphous and nanocrystalline regions, a grain boundary with 2-3 atom layer thickness exists. On the one hand, the nanocrystalline grains and their amorphous counterparts have very different band gap and band structures. As a result, they form heterojunction like structures in the interface regions. On the other hand, nanocrystalline grain acts like quantum dot. Free carriers can freely transport within grains, while transport to amorphous region is exhibited by quantum tunneling through the interface barrier. But the model may be modified. When the crystallinity of nc-Si:H films is far from 50\%, only the area ratio of amorphous region to nanocrystalline region and grain boundary thickness are different from that obtained from HQD model. In such case, as-deposited nc-Si:H can also be treated in terms of HQD model. Between nanocrystalline phase and amorphous phase a grain boundary barrier forms. In terms of generalized Bayesian theorem (GBT) model used in polycrystalline silicon, the amount of acceptor surface states in the grain boundary form due to the surface absorption of oxygen and other gas atoms. Acceptor surface is electrically exhausted and charged to create a potential energy barrier, which impedes the transport of free carriers from one grain to another. For polycrystalline silicon, the conduction band near boundary is convex [17], while the density of acceptor surface state of nc-Si:H is one order smaller than that of polycrystalline silicon [18]. It is obviously attributed to the fill-up of the acceptor surface state by the existing hydrogen in the boundary of nc-Si:H [19]. Evangelistic once suggested that the discontinuity of valence band near grain boundary for crystalline $\mathrm{Si} /$ amorphous $\mathrm{Si}(\mathrm{c}-\mathrm{Si} / \mathrm{a}-\mathrm{Si}$ ) heterojunction can be neglected due to the disorder atoms at the grain boundary, while the conduction band near grain boundary is concave-downward [20]. Wang et al. also discover the concave-down conduction band near grain boundary of nc-Si:H film [21]. It is differ-

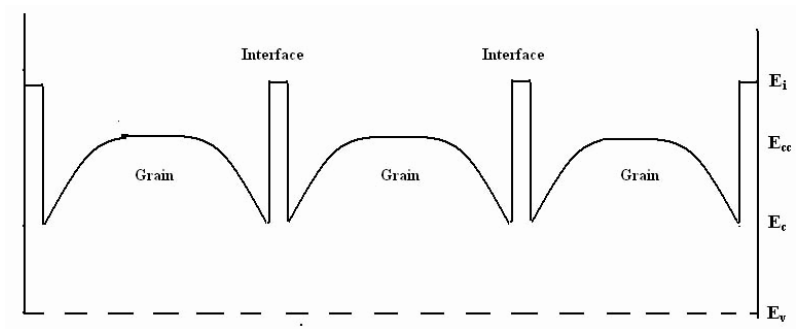

Fig. 6. Band sketch for nc-Si:H film in terms of HQD model.

ent from polycrystalline silicon. It may arise from the fillup of acceptor surface state by donor hydrogen. In terms of HQD model, the band gap of nc-Si:H film is sketched in Fig. 6, where $E_{\mathrm{v}}, E_{\mathrm{c}}, E_{\mathrm{cc}}$ and $E_{\mathrm{i}}$ correspond to the valence band top, conduction band bottom for nc-Si:H, conduction band bottom for c-Si and interface level. In terms of classical semiconductor theory, the carrier concentration can be determined by the following formula:

$$
n=n_{0} \exp \left(-E_{\mathrm{d}} / k T\right)
$$

where $E_{\mathrm{d}}, n$, and $T$ are activation energy, free carrier concentration, and measurement temperature, respectively. For as-deposited nc-Si:H films, their $E_{\mathrm{d}}$ (about 
0.4-0.5 eV) has a small difference, leading to a small difference between their free carrier concentrations at room temperature. According to classical semiconductor theory, dark electrical conductivity is described as follows:

$$
\sigma=n q \mu,
$$

where $\mu$ is the effective carrier mobility. The $\sigma$ seemingly varies a little for different as-deposited nc-Si:H films. However, as indicated in Fig. 5, obvious change of electrical conductivity of films takes place in the range of $10^{-2}$ to $10^{-5} \mathrm{~S} / \mathrm{cm}$, implying the carrier concentration is not the main factor for electrical conductivity. Classical semiconductor theory is not appropriate for nc-Si:H films. A mandatory correction is required. The modified electrical conductivity of nc-Si:H can be described by the following formula [16] in terms of HQD model:

$$
\sigma=n e \mu=\sigma_{0} \exp \left(-E_{d} / k T\right) F\left(\left\langle q^{2}\right\rangle, T\right),
$$

where $E_{\mathrm{d}}$ and $F\left(\left\langle q^{2}\right\rangle, T\right)$ are activation energy and quantum tunneling function, respectively. In terms with HQD model, free carrier can freely ballistically transport within grain, while transport to amorphous region is by quantum tunneling through interface barrier [16]. In terms of tunneling theory, the small interface thickness or interface barrier width helps the increase in quantum tunneling probability. In fact, the crystallinity determines the interface thickness. The larger crystallinity leads to the smaller interface thickness, which is the main reason for the increase in electrical conductivity with the crystallinity increasing. The free carrier concentration in amorphous regions is much smaller than in nanocrystalline region resulting from the quantum tunneling mechanism. On the other hand, the grain boundary can effectively diffract free carrier. Therefore, the effective carrier concentration can be greatly lowered due to the grain boundary and heterojunction between amorphous and nanocrystalline regions. The lowering effective carrier concentration can be regarded as the decreasing effective carrier mobility due to the carrier transport mechanism of nc-Si:H film. The effective carrier mobility rather than effective carrier concentration is the main effect factor for the dark electrical conductivity.

\section{Conclusions}

Microstructure and electrical properties of as-deposited nc-Si:H films on glass substrate at low temperature were characterized by average grain size, crystallinity and electrical conductivity data obtained from the Raman and electrical spectroscopy, respectively. The results of our investigation demonstrate that the deposition rate is nearly linear with $\mathrm{SC}$, which can be explained by the reversible reaction in formula (1). The average grain size, crystallinity and electrical conductivity of the $n c-S i: H$ films have a similar change with $T_{\mathrm{s}}$. A threshold $T_{\mathrm{s}}$ determined by SC can be observed in their corresponding spectroscopy vs. $T_{\mathrm{s}}$. The dependence of crystallinity, average grain size and electrical conductivity on $T_{\mathrm{s}}$ can be accounted for by surface diffusion model and heterojunction quantum dot model. The effective carrier mobility rather than free carrier concentration has the main effect on the dark electrical conductivity of as-deposited nc-Si:H films.

\section{Acknowledgments}

The work is supported by National Nature Science Foundation of China (grant No. 60807001) and National Basic Research Program of China (grant No. 2006CB202601).

\section{References}

[1] J. Meier, P. Torres, R. Platz, S. Dubail, U. Kroll, A.N. Selvan, N.P. Vauche, C. Hof, D. Fischer, A. Shar, K.D. Ufert, P. Giannoules, J. Koehler, Mater. Res. Soc. Symp. Proc. 420, 3 (1996).

[2] K. Yamamoto, M. Yoshimi, T. Suzuki, Y. Tawada, T. Okamoto, A. Nakajima, Mater. Res. Soc. Symp. Proc. 507, 131 (1998).

[3] K. Prasad, F. Finger, S. Dubail, A. Shah, M. Schubert, J. Non-Cryst. Solids 137, 681 (1991).

[4] R. Galloni, Renewable Energy 8, 400 (1996).

[5] C. Beneking, B. Rech, J. Foelsch, H. Wagner, Phys. Status Solidi B 194, 41 (1996).

[6] H. Wagner, Phys. Status Solidi B 192, 229 (1995).

[7] H. Takagi, H. Ogawa, Y. Yamazaki, A. Ishizaki, T. Nakagiri, Appl. Phys. Lett. 56, 2379 (1990).

[8] E. Fortunato, R. Martins, I. Ferrira, M. Santos, A. Magarico, L. Guimaraes, J. Non-Cryst. Solids 115, 120 (1989).

[9] T. Takagi, R. Hayashi, G. Ganguly, M. Kondo, A. Matsuda, Thin Solid Films 345, 75 (1999).

[10] J. Perrin, O. Leroy, M.C. Bordage, Contrib. Plasma Phys. 36, 3 (1996).

[11] S. Veprek, F.A. Sarott, Z. Iqbal, Phys. Rev. B 36, 3344 (1987).

[12] M. Jana, D. Das, A.K. Barua, Sol. Energy Mater. Sol. Cells 74, 407 (2002).

[13] T. Kaneko, K.T. Onisawa, M. Wakag, Y. Kita, T. Minemura, Jpn. J. Appl. Phys. 32, 4907 (1993).

[14] M. Akihisa, Thin Solid Films 337, 1 (1999).

[15] O. Tatusya, U. Osamu, N. Matsuhei, Surf. Sci. 458, 216 (2000).

[16] Y.L. He, M.B. Yu, G.Y. Hu, Q. Zhang, Acta Phys. Sinica 46, 1636 (1997).

[17] G. Harbeke, Polycrystalline Semiconductors, Springer-Verlag, New York 1985.

[18] Y.W. Seto, J. Appl. Phys. 46, 5247 (1975).

[19] M.B. Yu, Y.L. He, H.T. Liu, J.S. Luo, Acta Phys. Sinica 44, 634 (1995).

[20] F. Evangelistic, J. Non-Cryst. Solids 77-78, 969 (1985).

[21] F. Wang, H.N. Lin, Y.L. He, A. Schweiger, R. Schwarz, J. Non-Cryst. Solids 137-138, 511 (1991). 